\title{
Analysis of bit-stuffing codes and lower bounds on capacity for 2-D constrained arrays using quasistationary measures
}

\section{Forchhammer, Søren}

Published in:

Proceedings. International Symposium on Information Theory, 2004. ISIT 2004.

Link to article, DOI:

10.1109/ISIT.2004.1365199

Publication date:

2004

Document Version

Publisher's PDF, also known as Version of record

Link back to DTU Orbit

Citation (APA):

Forchhammer, S. (2004). Analysis of bit-stuffing codes and lower bounds on capacity for 2-D constrained arrays using quasistationary measures. In Proceedings. International Symposium on Information Theory, 2004. ISIT 2004. IEEE. https://doi.org/10.1109/ISIT.2004.1365199

\section{General rights}

Copyright and moral rights for the publications made accessible in the public portal are retained by the authors and/or other copyright owners and it is a condition of accessing publications that users recognise and abide by the legal requirements associated with these rights.

- Users may download and print one copy of any publication from the public portal for the purpose of private study or research.

- You may not further distribute the material or use it for any profit-making activity or commercial gain

- You may freely distribute the URL identifying the publication in the public portal 


\title{
Analysis of Bit-Stuffing Codes and Lower Bounds on Capacity for 2-D Constrained Arrays using Quasi-Stationary Measures
}

\author{
Søren Forchhammer \\ Research Center COM, 345v \\ Technical University of Denmark \\ DK-2800 Lyngby, Denmark \\ e-mail: sf@com.dtu.dk
}

\begin{abstract}
A method for designing quasi-stationary probability measures for two-dimensional (2-D) constraints is presented. This measure is derived from a modified bit-stuff coding scheme and it gives the capacity of the coding scheme. This provides a constructive lower bound on the capacity of the 2-D constraint. The main examples are checkerboard codes with binary elements. The capacity for one instance of the modified bit-stuffing for the 2-D runlengthlimited $\operatorname{RLL}(2, \infty)$ constraint is calculated to be $\mathbf{0 . 4 4 1 4}$ bits/symbol. For the constraint given by a minimum (1-norm) distance of 3 between 1s a code with capacity $0.3497 \mathrm{bits} / \mathrm{symbol}$ is given.

\section{INTRODUCTION}

We present a method for designing two-dimensional (2-D) constrained codes based on bit-stuffing. We consider 2-D arrays with elements taken from a finite alphabet. The constraint is specified by the set of admissible configurations on an $N$ by $M$ rectangle. For 2 -D RLL $(1, \infty)$, bit-stuffing provides efficient coding [1]. In [2] bit-stuffing for 2-D RLL $(d, \infty), d \geq 2$ were considered. We shall take a slightly different approach to bit-stuffing in order to facilitate analysis e.g. providing a constructive lower bound on the capacity of the constraint. The method is generally applicable to checkerboard constraints, where a 1 must be surrounded be a certain pattern of $0 \mathrm{~s}$, meaning that a 0 is always admissible.
\end{abstract}

\section{QUASI-STATIONARY MEASURES}

A quasi stationary measure may be introduced by concatenating arrays. Given a constraint, let $\mathbf{W}$ denote a stochastic variable defined on an $n$ by $m$ array, which may take on any configuration admissible by the constraint. Let $\mathbf{X}$ and $\mathbf{Z}$ denote variables representing the first and last $M-1$ columns (with $n$ elements). Let $\mathbf{Y}$ denote a variable representing the middle $m-2 M+2$ columns. We assume that the measures on the boundaries, $\mathbf{X}$ and $\mathbf{Z}$ are identical for the measures, $\mathbf{W}$ to be considered. Thus, starting with $\mathbf{X}_{0} \mathbf{Y}_{0} \mathbf{Z}_{0}$, arrays $\mathbf{Y}_{i} \mathbf{Z}_{i}$ may repeatedly be added to form $\mathbf{X}_{0}\left\{\mathbf{Y}_{j} \mathbf{Z}_{j}\right\}_{0}^{K}$, such that $\mathbf{Z}_{i-1} \mathbf{Y}_{i} \mathbf{Z}_{i}$ has the same measure as $\mathbf{W}$. The entropy (per symbol) is given by the conditional entropy of $\mathbf{Y}_{i} \mathbf{Z}_{i}$ given $\mathbf{Z}_{i-1}$ which is

$$
\frac{H_{W}(m)-H_{X}(M-1)}{m-M+1} .
$$

where $H_{W}(m)$ is the entropy of $\mathbf{W}$ (per row) and $H_{X}(M-1)$ is the entropy of $\mathbf{X}$ (per row). A simple way to specify $\mathbf{W}$ in (1) is to assign probabilities to the bit-stuffing scheme below. The boundaries $\mathbf{X}$ and $\mathbf{Z}$ are specified by identical but independent bit-stuffing schemes. The middle columns $\mathbf{Y}$ are specified by bit-stuffing conditional on the boundaries $\mathbf{X}$ and $\mathbf{Z}$.

\section{NumERICAL RESULTS}

Two examples with binary elements and constraint size $N=$ $M=3$ are considered. For the $\operatorname{RLL}(2, \infty)$ constraint, analysis of the modified bit-stuffing was carried out calcultating capacities, $C$ for $m=12$. The transition probabilities for a new row of $\mathbf{W}$ were determined by the products of (conditional) probabilities adressing and bit-stuffing the elements of the new line of $\mathbf{X}$ and $\mathbf{Z}$ before $\mathbf{Y}$ and using the same conditional probabilities for the corresponding elements of $\mathbf{X}$ and $\mathbf{Z}$. Thus the prerequisites for (1) is satisfied. Let $p_{1}$ denote the probabilities of writing a 1 when this is admissible. Simple bit-stuffing writing an unbiased sequence with $p_{1}=1 / 2$ gave $C=0.388$. Using a single biased sequence gave $C=0.437$ for optimal choice of $p_{1}$. Finally the values of $p_{1}$ may be chosen independently for each column of $\mathbf{X}$ and $\mathbf{Y}$. (The $p_{1}$ values of $\mathbf{Z}$ are given by $\mathbf{X}$.) This gave a best value of $C=0.44149$, also providing a lower bound for the constraint. This is a fair improvement on the lower bound of 0.4267 on the capacity of (diagonal) bit-stuffing in [2].

Capacities were also calculated for applying the modified bit-stuffing scheme to the constraint given by a min. (1-norm) distance of 3 between $1 \mathrm{~s}$. The results obtained for $m=15$ were $C=0.276$ when writing an unbiased sequence, $C=$ 0.344 for a single biased sequence and $C=0.3477$ choosing different biased sequences for each column of $\mathbf{X}$ and $\mathbf{Y}$. For this constraint the boundaries, $\mathbf{Z}_{i-1}$ and $\mathbf{Z}_{i}$ must be at least an additional row ahead in order to bit-stuff the elements of $\mathbf{X}$ and $\mathbf{Z}$ independently of past elements of $\mathbf{Y}$. A more elaborate scheme for specifying $\mathbf{W}$ in (1) was also devised. The probabilities $p_{1}$ were made dependent on the other elements on the $(N-1=) 2$ previous rows. The next row of $\mathbf{X}$ (and $\mathbf{Z}$ ) is specified by probabilities conditioned on the two previous rows. The new row of $\mathbf{Y}$ is specified by probabilities conditioned on 3 rows of $\mathbf{X}$ and $\mathbf{Z}$ and 2 rows of $\mathbf{Y}$. These conditional probabilities were obtained from the maxentropic solution [3] for $\mathbf{W}$ (with two rows forming the states). This gave a capacity of $C=0.3497$, which also provides a new lower bound for the constraint.

\section{REFERENCES}

[1] R.M. Roth, P.H. Siegel and J.K. Wolf, "Efficient coding schemes for the hard-square model," IEEE Trans. Inform. Theory, vol. 47, pp. 1166-76, March, 2001.

[2] S. Halevy, J. Chen, R.M. Roth, P.H. Siegel, J.K. Wolf, "Improved bit-stuffing bounds on two-dimensional constraints," Proc. ISIT'02, Lausanne, Switzerland, p. 385, 2002.

[3] S. Forchhammer and J. Justesen, "Entropy bounds for constrained two-dimensional random fields," IEEE Trans. Inform. Theory, vol. 45, no. 1, pp. 118-127, Jan. 1999. 